\title{
TITLE:
}

\section{Charge-carrier generation in organic solar cells using crystalline donor polymers.}

\section{$\operatorname{AUTHOR}(\mathrm{S})$ :}

Tamai, Yasunari; Tsuda, Kazuki; Ohkita, Hideo; Benten, Hiroaki; Ito, Shinzaburo

\section{CITATION:}

Tamai, Yasunari ... [et al]. Charge-carrier generation in organic solar cells using crystalline donor polymers.. Physical chemistry chemical physics 2014, 16(38): 20338-20346

\section{ISSUE DATE:}

2014-06-19

URL:

http://hdl.handle.net/2433/200182

\section{RIGHT:}

This journal is (c) the Owner Societies 2014.; This is not the published version. Please cite only the published version.; この論文は出版社版で ありません。引用の際には出版社版をご確認ご利用ください。 


\title{
ARTICLE
}

Cite this: DOI: $10.1039 /$ xoxxooooox

\section{Charge-Carrier Generation in Organic Solar Cells with Crystalline Donor Polymers}

\author{
Yasunari Tamai, ${ }^{a}$ Kazuki Tsuda, ${ }^{a}$ Hideo Ohkita,${ }^{a, b}$ Hiroaki Benten ${ }^{a}$ and \\ Shinzaburo Ito $^{a}$
}

Received ooth January 2012, Accepted ooth January 2012

DOI: $10.1039 /$ xoxxooooox

www.rsc.org/

\begin{abstract}
Charge generation and recombination dynamics in a blend film of crystalline low-bandgap polymer, poly[(4,4-bis(2-ethylhexyl)dithieno[3,2-b:2',3'-d]silole)-2,6-diyl-alt-(4,7-bis(2thienyl)-2,1,3-benzothiadiazole)-4,7-diyl] (PSBTBT), and [6,6]-phenyl-C61-butyric acid methyl ester (PCBM) were studied by transient absorption spectroscopy. Upon photoexcitation of PSBTBT absorption band at $800 \mathrm{~nm}$, singlet excitons were promptly generated, and then rapidly converted into polarons in a few picoseconds. We found that there are two different polarons in PSBTBT: one is ascribed to polarons generated in disorder phase and the other is ascribed to polarons in crystalline phase. On a time scale of nanoseconds, $\sim 50 \%$ of polarons in the disorder phase recombined geminately to the ground state. On the other hand, such geminate recombination was negligible for polarons in the crystalline phase. As a result, the overall charge dissociation efficiency is as high as $\sim 75 \%$ for PSBTBT/PCBM blend films. On the basis of these analyses, we discuss the role of polymer crystallinity on the charge-carrier generation in organic solar cells.
\end{abstract}

\section{Introduction}

Charge-carrier generation at the interface of donor polymer and acceptor fullerene materials is one of the key issues to be solved for developing highly efficient organic solar cells. ${ }^{1-9}$ Upon photoexcitation of either materials, singlet excitons are promptly generated and then diffuse to the donor/acceptor interface within their short lifetimes. As a result of charge transfer (CT) at the interface, polymer polarons and fullerene anions are generated with high efficiency. In some blends, almost all the polymer polarons and fullerene anions can be dissociated into free charge carriers to be collected as photocurrent efficiently. In other blends, on the other hand, a part of polymer polarons and fullerene anions are still Coulombically bound to form interfacial CT states, which are likely to recombine to the ground state and hence limit the device performance. At this moment, little is known about the origin of these different charge generation mechanisms.

Recent studies have shown that the charge-carrier generation is dependent on crystallinity of donor polymers. ${ }^{10-14}$ For example, we have previously reported different charge dissociation efficiency in blends of poly(3-hexylthiophene) (P3HT) and [6,6]-phenyl-C61-butyric acid methyl ester (PCBM). In blend films of regiorandom P3HT (RRa-P3HT, the chemical structure is shown in Figure 1e) and PCBM (Figure 1c), the charge-carrier generation efficiency is as low as $\sim 30 \%$. ${ }^{6,10}$ This is probably because PCBM molecules are likely to disperse homogeneously in RRa-P3HT amorphous films. ${ }^{15-17}$ In blend films of regioregular P3HT (RR-P3HT, Figure 1d) and PCBM, $>90 \%$ of polarons can be dissociated into free charge carriers. This is partly because RR-P3HT self-organizes into two- dimensional $\pi$-stacked lamellar structures to form crystalline fibrils. ${ }^{18-22}$ The difference in the charge-carrier generation is in good agreement with the difference in the external quantum efficiency (EQE) of the two devices: $<5 \%$ for RRaP3HT/PCBM ${ }^{17}$ and $>80 \%$ for RR-P3HT/PCBM. ${ }^{23-25}$ A similar crystallinity dependence has been reported for two typical lowbandgap polymers, poly[(4,4-bis(2-ethylhexyl)-4Hcyclopenta[2,1-b;3,4-b']-dithiophene)-2,6-diyl-alt-(2,1,3benzothiadiazole)-4,7-diyl] (PCPDTBT, Figure 1b) and poly[(4,4-bis(2-ethylhexyl)dithieno[3,2-b:2',3'-d]silole)-2,6diyl-alt-(2,1,3-benzothiadiazole)-4,7-diyl] (PSBTBT, Figure 1a). Without solvent additives, PCPDTBT/PCBM blends form amorphous films and hence exhibit relatively low PCE of $\sim 3 \%$. ${ }^{26}$ With solvent additives such as 1,8-diiodooctane (DIO) or octanedithiol (ODT), polymer chain packing is promoted, resulting in a PCE of as high as 5.5\%. ${ }^{27,28}$ On the other hand, PSBTBT/PCBM blends self-organize into $\pi$-stacked crystals in thin films, resulting in a PCE of $>5 \%$ without solvent additives, and 5.6\% after thermal annealing. ${ }^{29-34}$ All these reports suggest that polymer crystallinity can promote charge-carrier generation effectively. However, it is still not fully understood how polymer crystallinity impacts on the charge-carrier generation.

Herein, we study the charge generation and recombination dynamics in blends of crystalline donor polymer PSBTBT and acceptor PCBM by transient absorption spectroscopy. Each efficiency is quantitatively discussed for the following fundamental photovoltaic conversion processes: the exciton diffusion to a donor/acceptor interface $\left(\eta_{\mathrm{ED}}\right)$, the charge transfer at the interface $\left(\eta_{\mathrm{CT}}\right)$, and the charge dissociation into free charge carriers $\left(\eta_{\mathrm{CD}}\right)$. On the basis of these findings with previous reports, we 
Figure 1. Chemical structures of a) PSBTBT, b) PCPDTBT, c) PCBM, d) RR-P3HT, and e) RRa-P3HT. f) Absorption spectra of PSBTBT films prepared from o-dichlorobenzene (black solid line) and chloroform (gray solid line), respectively. The broken line represents the absorption spectrum of PSBTBT in a 1-chloronaphthalene solution.

comprehensively discuss the relevance of polymer crystallinity to the charge generation dynamics.

\section{Results}

\section{Absorption Spectra}

Figure 1f shows the absorption spectra of PSBTBT pristine films and PSBTBT in a 1-chloronaphthalene solution, which is a good solvent for PSBTBT. As shown in the figure, PSBTBT in a 1-chloronaphthalene solution exhibits a broad absorption band without a vibrational structure at around $650 \mathrm{~nm}$, suggesting that polymers are well dissolved in the solvent. On the other hand, PSBTBT films exhibit a characteristic absorption shoulder at around $750 \mathrm{~nm}$, which is attributable to the absorption band of crystalline PSBTBT as reported previously. ${ }^{31}$ The absorption shoulder is more pronounced for the PSBTBT film prepared from $o$-dichlorobenzene than that from chloroform, suggesting that the former film has higher crystallinity than the latter one. This is because $o$ dichlorobenzene (b.p. $180.5{ }^{\circ} \mathrm{C}$ ) would be more slowly evaporated during the spincoating and hence promote the crystallization of PSBTBT more effectively than chloroform (b.p. $61.2^{\circ} \mathrm{C}$ ).

\section{Transient Absorption Spectra}

Figure 2 shows the transient absorption spectra of PSBTBT pristine (broken line) and PSBTBT/PCBM blend (50 : $50 \mathrm{wt} \%$, solid lines) films prepared from $o$-dichlorobenzene. The excitation wavelength was set at $800 \mathrm{~nm}$ to excite PSBTBT domains selectively. In other words, we focus on the charge generation dynamics from PSBTBT singlet excitons in this study. For the PSBTBT pristine film, as shown by the broken line, a large absorption band was observed at around $1500 \mathrm{~nm}$, and decayed monotonically with time. This band is attributable to the absorption of PSBTBT singlet excitons as reported previously. ${ }^{35}$ The singlet exciton band in the film was redshifted compared to that in a 1-chloronaphthalene solution (data is shown in the Supplementary Information), suggesting that singlet excitons in thin films are not localized onto single chains but delocalized over the crystalline phase as will be discussed later. ${ }^{36,37}$ Note that the negative signals at the visible region are assigned to the ground state photobleaching of PSBTBT. For PSBTBT/PCBM blend films, as shown by the red line, the singlet exciton band was also observed but already decayed even at 0 ps compared to that observed for PSBTBT pristine films. Instead, a small absorption tail was observed

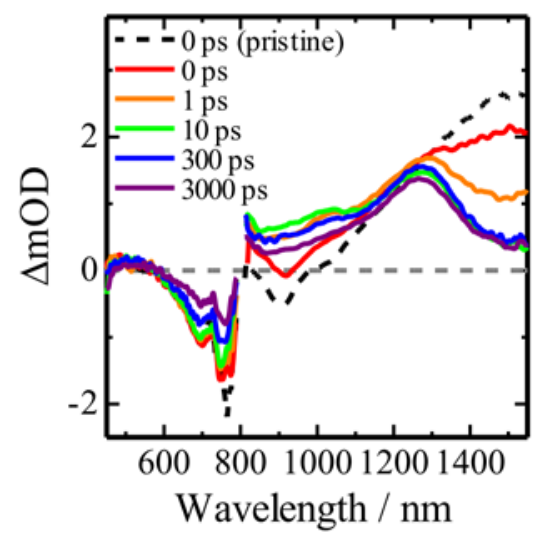

Figure 2. Transient absorption spectra of a PSBTBT/PCBM blend film measured at 0 (red), 1 (orange), 10 (green), 300 (blue), and $3000 \mathrm{ps}$ (purple) after the laser excitation. The film was excited at $800 \mathrm{~nm}$ with a fluence of $11 \mu \mathrm{J} \mathrm{cm}^{-2}$. The broken line represents the $\mathrm{S}_{1}-\mathrm{S}_{\mathrm{n}}$ absorption band observed for a PSBTBT pristine film. 
from 800 to $1300 \mathrm{~nm}$. These findings suggest that a part of singlet excitons are converted into other species even within a short duration of the excitation pulse ( $\sim 100 \mathrm{fs})$. The singlet exciton absorption signals at $1500 \mathrm{~nm}$ rapidly decayed in a few picoseconds. Instead, the broad absorption tail from 800 to $1300 \mathrm{~nm}$ became more pronounced with time. At $10 \mathrm{ps}$ after the laser excitation (green line), the singlet exciton band completely disappeared, and broad absorption bands were observed at around 1000 and $1300 \mathrm{~nm}$. Subsequently, the band at $1000 \mathrm{~nm}$ decayed slowly while the band at $1300 \mathrm{~nm}$ remained almost the same. This finding suggests that these two absorption bands can be ascribed to different species. As shown in Figure 3, both bands were still observed even on a time scale of microseconds, and not identical to PSBTBT triplet excitons, which have an absorption band at $1100 \mathrm{~nm}$ as shown in the Supplementary Information. Thus, these bands are ascribed to PSBTBT polarons.

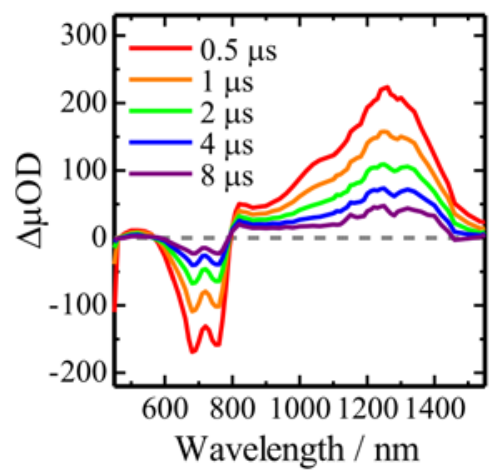

Figure 3. Transient absorption spectra of a PSBTBT/PCBM blend film measured at $0.5,1,2,4$, and $8 \mu$ s after the laser excitation from top to bottom. The film was excited at 400

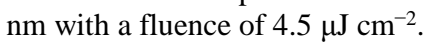

In order to assign these polaron bands, we measured the transient absorption spectra of PSBTBT/PCBM blend films with different crystallinity. As shown in Figure 4, the polaron band at $1000 \mathrm{~nm}$ was more pronounced for the blend film prepared from chloroform than that from o-dichlorobenzene. Furthermore, as shown in the Supplementary Information, the absorption band was observed only at $1000 \mathrm{~nm}$ for PSBTBT in

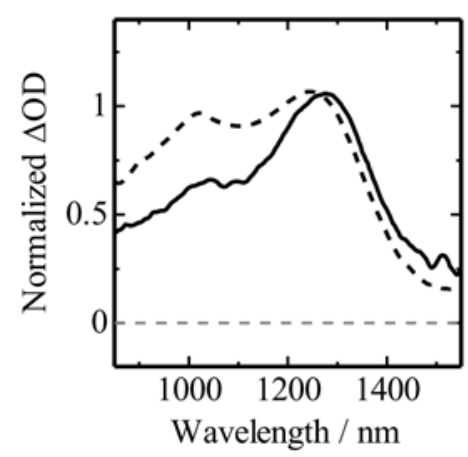

Figure 4. Normalized transient absorption spectra of PSBTBT/PCBM blend films prepared from odichlorobenzene (solid line) and chloroform (broken line) measured at $10 \mathrm{ps}$ after the laser excitation. a 1-chloronaphthalene solution blended with PCBM. We therefore assign the band at $1000 \mathrm{~nm}$ to polarons generated in PSBTBT disorder phase and the band at $1300 \mathrm{~nm}$ to polarons in PSBTBT crystalline phase. Note that no absorption of PCBM anions is observed because of its small absorption coefficient. ${ }^{38}$ In summary, upon the photoexcitation at $800 \mathrm{~nm}$, the primary photoexcitation in PSBTBT/PCBM blend films is delocalized PSBTBT singlet excitons, and then rapidly converted into polarons. There are two different polarons in the blend film: one is polarons generated in disorder phase and the other is polarons in crystalline phase. The disorder polarons decayed slowly on a time scale of nanoseconds, but the crystalline polarons remained almost the same. Figure 5 summarizes the absorption spectra of these transients (see the Supplementary Information).

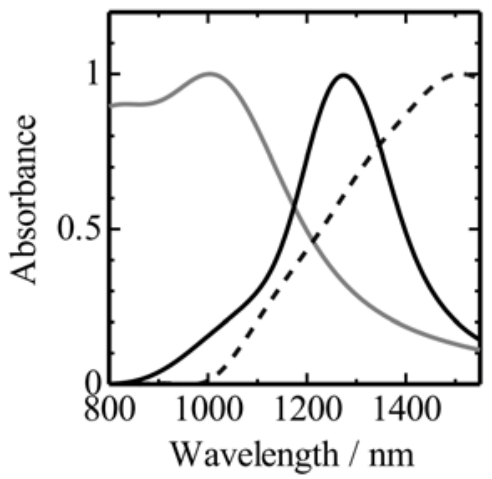

Figure 5. Normalized absorption spectra of PSBTBT singlet excitons (broken line), PSBTBT polarons in disorder phase (gray solid line), and PSBTBT polarons in crystalline phase (black solid line).

\section{Transient Absorption Decays}

To discuss the formation and decay dynamics of polarons in more detail, we carefully analysed the time evolution of transient species on the basis of spectral simulation by using absorption templates as shown in Figure 5. The transient absorption spectra observed can be well reproduced by a sum of these templates (some examples of reproduced spectra are shown in the Supplementary Information). Figure 6a shows the time evolution of singlet excitons (open circles), polarons in disorder phase (open triangles), and polarons in crystalline phase (open squares) in the picosecond time domain. For the quantitative discussion, $\triangle \mathrm{OD}$ was converted into the number density $N(t)$ according to the Beer-Lambert law: $N(t)=\Delta \mathrm{OD}(t)$ $N_{\mathrm{A}}(1000 \varepsilon l)^{-1}$ where $N_{\mathrm{A}}$ is the Avogadro's constant, $\varepsilon$ is the molar absorption coefficient (see the Supplementary Information), and $l$ is the film thickness. The closed circles represent the total population of these transients. Here, the number density of these transients is normalized by the total population at $0 \mathrm{ps}$. The rapid singlet exciton decay is well fitted with an exponential function with a lifetime of 0.8 ps (black solid line). On the other hand, the time evolution of two polarons can be well fitted with the same exponential rise functions (blue and red lines), indicating that polarons were generated from singlet excitons on a time scale of $<1 \mathrm{ps}$. Note that some polarons were promptly generated even at 0 ps. The 
total population was almost the same at this early time stage, suggesting that almost all excitons generated are converted into polarons $\left(\eta_{\mathrm{ED}} \times \eta_{\mathrm{CT}}=\sim 1\right)$. This is in good agreement with the efficient quenching of PSBTBT fluorescence as shown in the Supplementary Information.

Figure $6 \mathrm{~b}$ shows the time evolution of two polarons ranging from 10 to 2500 ps. Interestingly, polarons in crystalline phase still increased slightly even after singlet excitons completely disappeared. This rise component was well fitted with the sum of an exponential function with a lifetime of 24 ps and a constant fraction. To address the origin of the increase in the polarons in crystalline phase, we performed singular value decomposition (SVD) and global fitting analysis for the transient absorption spectra. ${ }^{39,40}$ Using SVD, the 3D matrix of
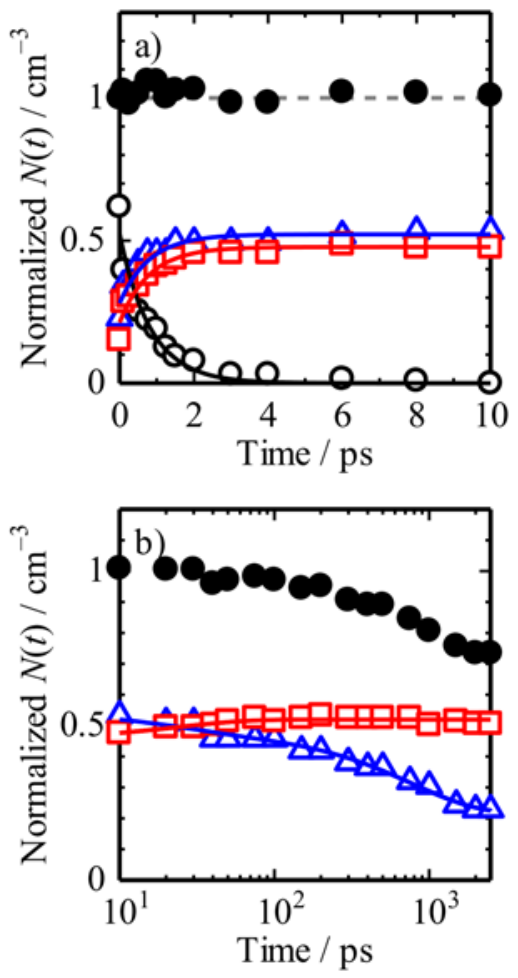

Figure 6. Time evolutions of singlet excitons (open circle), polarons in disorder phase (open triangles), and polarons in crystalline phase (open squares) in a PSBTBT/PCBM blend film prepared from $o$-dichlorobenzene on a time scale from pico- to nanoseconds. The closed circles represent the total population of these transient species. (a) The singlet exciton decay was fitted with an exponential decay function: $N(t)=\mathrm{A}_{\mathrm{d}}$ $\exp \left(-t / \tau_{\mathrm{d}}\right)$. The time evolution of polarons was fitted with the sum of an exponential rise and a constant fraction: $N(t)=\mathrm{A}_{r}$ $\exp \left(-t / \tau_{\mathrm{r}}\right)+B$. The time constant was the same as that of singlet exciton decay. (b) The time evolution of polarons in crystalline phase was fitted with the sum of an exponential rise and a constant fraction: $N(t)=\mathrm{A}_{\mathrm{r}} \exp \left(-t / \tau_{\mathrm{r}}\right)+\mathrm{B}$. The time evolution of polarons in disorder phase was fitted with the sum of two exponential decays and a constant fraction: $N(t)=$ $\sum A_{d} \exp \left(-t / \tau_{\mathrm{d}}\right)+B$. The shorter lifetime and its magnitude were the same as those of rise for polarons in crystalline phase. The solid lines represent the best fitting curves. the transient absorption data can be decomposed into its principal spectra and kinetic traces. The principal kinetic traces are then globally fitted to a multi-exponential function convoluted with the Gaussian instrument response function ( 130 fs). The pre-exponential coefficients are obtained as a function of the wavelength from fitting of the 3D matrix to the exponentials. Although it has no physical meaning to use multi-exponential functions, this analysis provides us an excellent initial clue to obtain insights into the dynamics of transient species. Applying this procedure to the PSBTBT/PCBM blend film, we obtained four exponential components and a constant component.

Figure 7 shows one of the pre-exponential coefficient spectra with a time constant of $19.3 \pm 250$ ps where the positive and negative signals correspond to decay and rise dynamics of the transient signal at the wavelength, respectively. As shown in the figure, the pre-exponential coefficient spectrum can be well reproduced by the sum of the positive spectrum of polarons in disorder phase (decay) and the negative spectrum of polarons in crystalline phase (rise). This finding suggests that polarons in crystalline phase increase with decreasing polarons in disorder phase. We therefore assign this rise and decay dynamics to hole transfer from disorder to crystalline phase as will be discussed later. As shown in Figure 6b, the decay dynamics of polarons in disorder phase was fitted with the sum of two exponential functions and a constant fraction. The shorter lifetime (fraction) was fixed to 24 ps (12\%), which is the same as that of the crystalline polaron rise. The longer lifetime (fraction) was evaluated to be 780 ps (49\%). Note that the other pre-exponential coefficient spectra are consistent with our assignments mentioned above (see the Supplementary Information).

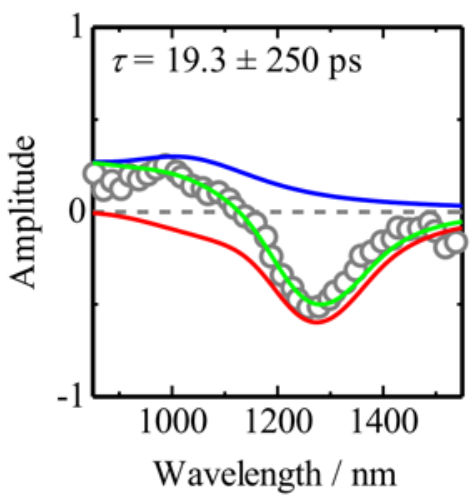

Figure 7. Pre-exponential coefficient spectrum (open circles) obtained by SVD and global fitting analysis. The time constant was $19.3 \pm 250$ ps. The blue and red lines represent spectral templates of PSBTBT polarons in disorder and crystalline phase, respectively. The green line represents the simulated spectrum by the sum of the blue and red lines.

As shown in Figure 8, the decay dynamics of polarons in disorder phase measured at $900 \mathrm{~nm}$ was independent of the excitation intensity at least $<25 \mu \mathrm{J} \mathrm{cm}^{-2}$, suggesting that polarons in disorder phase decayed monomolecularly. The decay lifetime was 780 ps, which is in good agreement with a CT emission lifetime of $\sim 810$ ps evaluated by the time- 


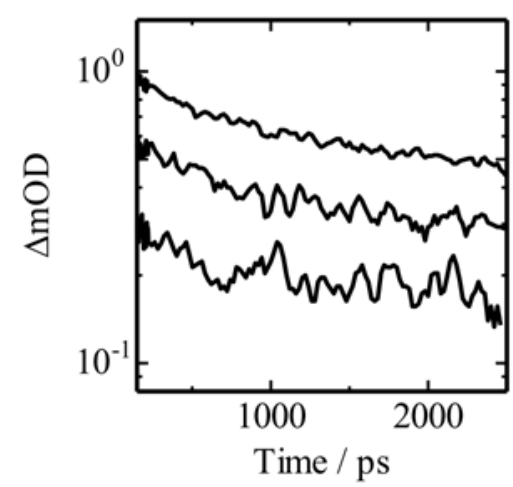

Figure 8. Transient absorption decays of a PSBTBT/PCBM blend film prepared from $o$-dichlorobenzene measured at 900 $\mathrm{nm}$. The excitation intensity was varied over 6,11 , and $25 \mu \mathrm{J}$ $\mathrm{cm}^{-2}$ from bottom to top.

correlated single-photon-counting (TCSPC) method (data is shown in the Supplementary Information). This agreement indicates that about a half of polarons in disorder phase recombine geminately to the ground state as will be discussed later. On the other hand, such geminate recombination was negligible for polarons in crystalline phase in this time domain as will be discussed later.

In summary, about a half of polarons are generated in PSBTBT disorder phase, and the others are in crystalline phase. The charge dissociation efficiency is almost unity for polarons in crystalline phase ( $\eta \mathrm{CD}^{\text {cry }}=\sim 1$ ), while it is as low as half for polarons in disorder phase $\left(\eta_{\mathrm{CD}}{ }^{\mathrm{dis}}=\sim 0.5\right)$. A part of polarons in disorder phase are transferred to crystalline phase with a time constant of 24 ps as will be discussed later. Consequently, as shown by the closed circles in Figure 6, the overall charge dissociation efficiency $\eta_{\mathrm{CD}^{\text {total }}}$ is as high as $\sim 0.75$ in PSBTBT/PCBM blends.

\section{Discussion}

For comparison, we first summarize efficiencies of each photovoltaic conversion process in PSBTBT/PCBM blend films estimated in this study along with those taken from some references ${ }^{10,12,20-22,41-43}$ in Table 1 . The efficiency of the exciton diffusion to the donor/acceptor interface ( $\left.\eta_{\mathrm{ED}}\right)$ is almost unity except for RR-P3HT/PCBM. For RR-P3HT/PCBM, $\eta_{\text {ED }}$ has been reported to be $\sim 0.95$ before the thermal annealing and $\sim 0.9$ after the thermal annealing. The efficiency of the charge transfer at the interface is highly efficient $\left(\eta_{\mathrm{CT}}=\sim 1\right)$ for all the blends mentioned here, suggesting that it is primarily dependent on the energetics of materials employed rather than polymer crystallinity. The efficiency of the charge dissociation into free carriers $(\eta \mathrm{CD})$ is clearly dependent on blend morphology. For amorphous polymers such as RRa-P3HT/PCBM and PCPDTBT/PCBM (w/o solvent additives) blends, the charge dissociation efficiency $\eta \mathrm{CD}$ is as low as $\sim 0.3^{10}$ and $\sim 0.5^{12}$, respectively. For more ordered blends of PCPDTBT/PCBM fabricated with solvent additives, the efficiency $\eta_{\mathrm{CD}}$ is improved to $\sim 0.7 .^{12,41}$ No clear diffraction peaks corresponding to (010) crystallographic plane have been reported for PCPDTBT/PCBM blend films with or without solvent additives, suggesting that crystallinity of PCPDTBT is much lower than that of PSBTBT ${ }^{30-33,42}$ and RR-P3HT. ${ }^{18-22}$ For crystalline polymers such as PSBTBT/PCBM and RR-P3HT/PCBM blends, on the other hand, the charge dissociation efficiency is almost unity for the crystalline phase $\left(\eta_{\mathrm{CD}^{\mathrm{cry}}}\right)$ but not so high for the disorder phase $\left(\eta \mathrm{CD}^{\mathrm{dis}}\right)$. For RR-P3HT/PCBM blends, $\eta \mathrm{CD}^{\text {dis }}$ is improved from $\sim 0.4$ to $\sim 0.7$ after the thermal annealing. As a result, the overall charge dissociation efficiency $\eta_{\mathrm{CD}}{ }^{\text {total }}$ is as high as more than 0.9. As summarized in Table 1, the dissociation efficiency $\eta \mathrm{CD}$ is not correlated with the $\pi-\pi$ stacking distance $d$, which is almost the same in these two crystalline blends, but rather correlated with the crystalline size in the $\pi$-stacking direction $L_{010}$ : it is reported to be $\sim 4.6 \mathrm{~nm}$ for $\mathrm{PSBTBT}_{\mathrm{PCBM}}{ }^{42}$ and $\sim 5.7 \mathrm{~nm}^{22}$ and $\sim 12 \mathrm{~nm}^{21}$ for RRP3HT/PCBM before and after the thermal annealing, respectively. We will discuss this correlation between $\eta \mathrm{CD}^{\text {total }}$ and $L_{010}$ later.

Exciton Harvesting and Charge Generation

Table 1. Each Efficiency of Polymer/PCBM Solar Cells.

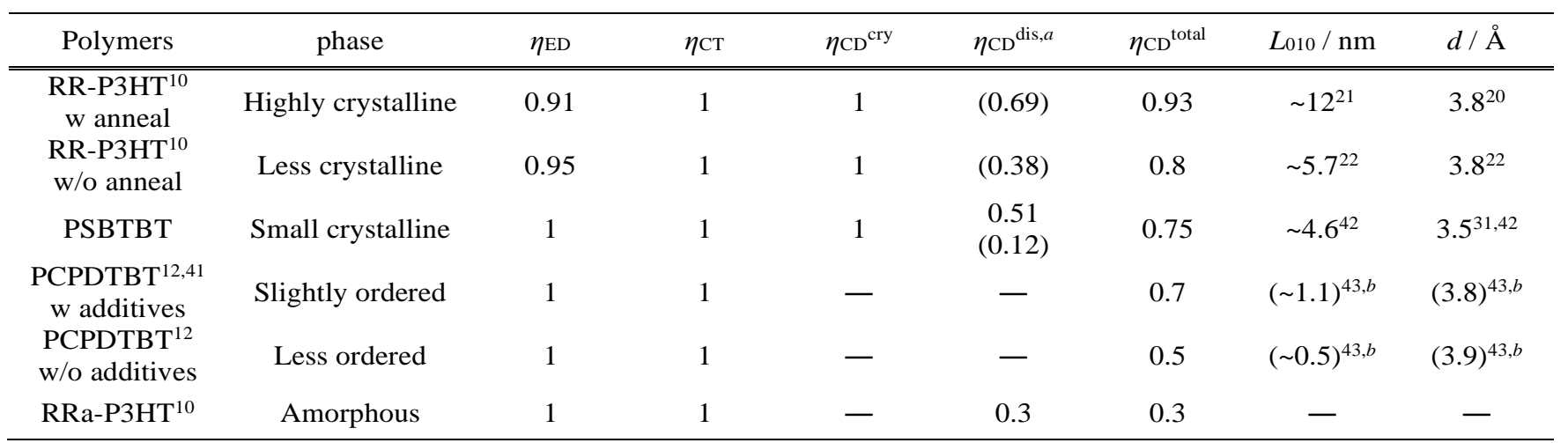

${ }^{a}$ The value in parenthesis is the charge dissociation efficiency by the hole transfer.

${ }^{b}$ This value was obtained from PCPDTBT pristine films. No clear diffraction peaks have been reported for PCPDTBT in the blend films, suggesting that PCPDTBT forms less ordered aggregates in the blend films. 
In PSBTBT/PCBM crystalline blend films, as shown in Figure $6 \mathrm{a}$, polarons are promptly generated not only in the disorder phase but also in the crystalline phase within a picosecond. This is in sharp contrast to RR-P3HT/PCBM crystalline blend films. In RR-P3HT/PCBM blend films, there are two pathways for the polaron generation: one is prompt charge generation at the interface, and the other is delayed charge generation following after the exciton diffusion to the interface in crystalline domains. The time constant of the delayed charge generation is as long as tens of picoseconds, which is dependent on the P3HT crystalline domain size. ${ }^{10,44}$ Upon the selective excitation of P3HT crystalline domains, the prompt charge generation is negligible and the delayed charge generation is dominant. Therefore, the prompt charge generation in PSBTBT/PCBM crystalline blend films is rather similar to that reported for PCPDTBT/PCBM and RRa-P3HT/PCBM amorphous blend films. ${ }^{10,12,41}$ The prompt charge generation in a picosecond indicates that no exciton diffusion is needed to reach PCBM molecules. This is probably because PCBM molecules are likely to be dispersed homogeneously in amorphous films. Indeed, the intermolecular distance of PCBM is estimated to be less than $1 \mathrm{~nm}$ at a PCBM fraction of $50 \mathrm{wt} \%$ assuming completely homogeneous distribution of PCBM molecules. ${ }^{45}$ However, PCBM in PSBTBT/PCBM blend films cannot be distributed homogeneously because of PSBTBT crystalline phase. We therefore speculate that PSBTBT crystalline domains are as small as PSBTBT singlet excitons. Indeed, a recent study has shown that the crystalline size $L_{010}$ is evaluated to be $\sim 4.6 \mathrm{~nm},{ }^{42}$ which would be comparable to PSBTBT singlet exciton size because it has been reported to be about 3-4 nm for P3HT and PCPDTBT. ${ }^{10,41,46}$ On the other hand, much large crystalline polymer domains with $L_{010} \sim 12$ $\mathrm{nm}$ have been reported for RR-P3HT/PCBM blend films. ${ }^{22}$ In such large domains, singlet excitons should diffuse to reach the heterojunction. Because of the large crystalline size of P3HT, $\sim 10 \%$ of excitons cannot reach the interface during their short lifetime. In other words, $\eta_{\mathrm{ED}}$ of RR-P3HT/PCBM is limited to $\sim 0.9,{ }^{10}$ which is lower than that of the other blends $\left(\eta_{\mathrm{ED}}=\sim 1\right)$.

a)

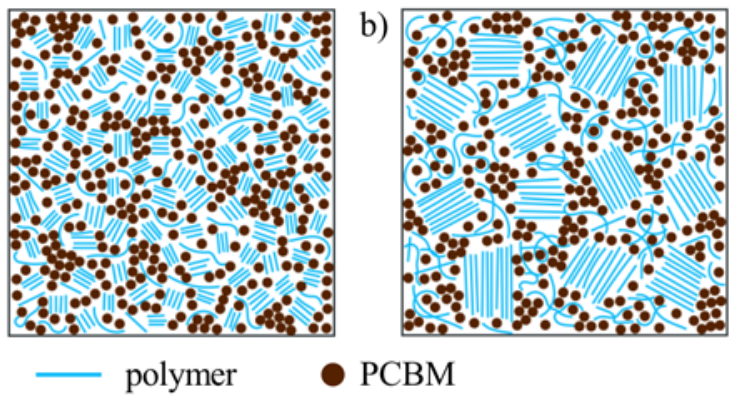

Figure 9. Schematic morphologies of a) PSBTBT/PCBM and b) RR-P3HT/PCBM blend films.

In summary, as shown in Figure 9a, PSBTBT forms small nanocrystals with a size of several nanometers in the blend films, which would be comparable to PSBTBT singlet excitons. As a result, all the PSBTBT excitons are quantitatively converted to polarons without the exciton diffusion $(\eta \mathrm{ED}=$ 1). ${ }^{14,47,48}$ This is in sharp contrast to RR-P3HT/PCBM blends: $\sim 10 \%$ of singlet excitons cannot reach the interface $\left(\eta_{\mathrm{ED}}=\sim 0.9\right)$ because of relatively large crystalline domains $\left(L_{010}=\sim 12 \mathrm{~nm}\right)$, as shown in Figure 9b. In other words, both PSBTBT and RR-
P3HT form crystalline phase in the blend films, but the film morphology is substantially different. The smaller crystalline domains in PSBTBT/PCBM blend films is beneficial for the efficient exciton diffusion to the heterojunction to generate the photocurrent.

\section{Hole Transfer}

We next focus on the hole transfer from disorder phase to crystalline phase in PSBTBT/PCBM blend films. A similar hole transfer has been reported for RR-P3HT/PCBM blend films. ${ }^{10}$ The driving force of such hole transfer is probably due to the potential energy cascades between pure crystalline phase and interfacial disorder phase where polymers and PCBM are molecularly mixed. ${ }^{13,48,49}$ In general, ionization potential (IP) of polymer films is reduced with increasing polymer crystallinity. For example, the IP of crystalline P3HT is estimated to be $4.6 \mathrm{eV}$ while that of disorder P3HT is $4.9 \mathrm{eV} .{ }^{50}$ As shown in the Supplementary Information, the IP of PSBTBT films is also reduced with increasing crystallinity: the IP of PSBTBT prepared from $o$-dichlorobenzene is estimated to be $4.89 \mathrm{eV}$, which is lower by $\sim 0.06 \mathrm{eV}$ than that from chloroform $(4.95 \mathrm{eV})$. We therefore conclude that some of polarons can be dissociated into free carriers by hole transfer from disorder to crystalline phase as shown in Scheme 1 . The charge dissociation efficiency by the hole transfer from disorder to crystalline phase is estimated to be 0.12 in PSBTBT/PCBM as mentioned above, which is lower than that in RR-P3HT/PCBM blend films ( 0.38 for before thermal annealing). Interestingly, the hole transfer efficiency in RR-P3HT/PCBM blend films increases from 0.38 to 0.69 after thermal annealing, indicating that the hole transfer is strongly dependent on the polymer crystallinity. This is probably because that the hole transfer efficiency depends on the energy offset between crystalline and

Scheme 1. a) Energetic Diagram and b) Blend Morphology of the Interface between Crystalline Polymer and Fullerene ${ }^{a}$

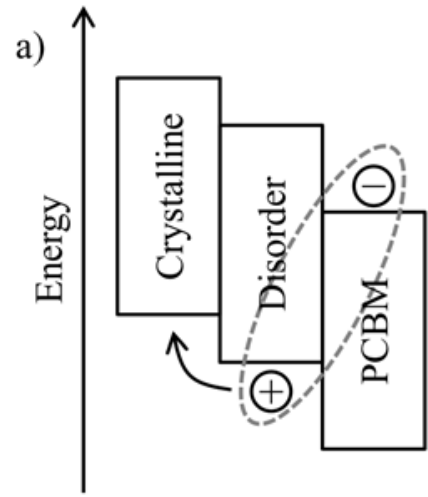

b)

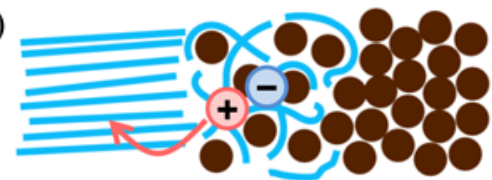

a Promptly generated polarons in disorder phase, which are tightly bound to PCBM anions, are transferred to crystalline phase promoted by an energy offset between disorder and crystalline phase. 
disorder phases. Owing to the higher crystallinity of RR-P3HT, the energy offset in RR-P3HT is larger than that in PSBTBT, resulting in more efficient hole transfer. On the other hand, the hole transfer is less efficient and hence the overall charge dissociation efficiency $\eta \mathrm{CD}^{\text {total }}$ is limited to $\sim 0.75$ in PSBTBT/PCBM blend films. In other words, more efficient hole transfer would result in further improvement in $\eta \mathrm{CD}^{\text {total }}$.

\section{Charge-Carrier Generation in Crystalline Phase}

We finally discuss the mechanism of efficient charge-carrier generation observed in crystalline phase. As shown in Figure $6 \mathrm{~b}$ (open squares), almost all polarons can be dissociated into free carriers in crystalline phase. Highly efficient chargecarrier generation is also observed for RR-P3HT/PCBM blend films. ${ }^{10}$ This is surprisingly high from the view point of conventional charge dissociation model based on the BraunOnsager pictures. ${ }^{51-53}$ Because of low dielectric constants of organic materials $\left(\varepsilon_{\mathrm{r}}=\sim 3-4\right)$, an electron on an acceptor and a hole on a donor are tightly bound at the interface by the strong Coulomb attraction, and hence efficient charge dissociation would require large driving force such as strong electric field of $>10^{8} \mathrm{~V} \mathrm{~m}^{-1} .{ }^{54,55}$ However, this is not the case with efficient organic solar cells as mentioned above and reported by other researchers. On the other hand, as mentioned above, we found a positive correlation that $\eta_{\mathrm{CD}}{ }^{\text {total }}$ increases with increasing $L_{010}$. We therefore speculate that delocalization of hole polarons plays a key role in the efficient charge-carrier generation.

The importance of such delocalization has been discussed by several reports including ours. ${ }^{10,41,56-59}$ We have previously proposed that more delocalized excitons in RR-P3HT/PCBM are likely to form more delocalized polarons and hence more efficiently be dissociated into free carriers than in RRaP3HT/PCBM. ${ }^{10}$ Deibel and his co-workers performed kinetic Monte Carlo simulations for hopping transport of Coulombically bound charges, and suggested that delocalization of hole polarons along polymer chains reduces the Coulomb attraction and increases dissociation efficiency. ${ }^{56}$ They also pointed out that polymer crystallization may result in further improvement in the dissociation efficiency. This is consistent with our observations. In particular, we emphasize the importance of polymer crystallization because the charge dissociation efficiency in crystalline PSBTBT is much higher than that of disorder PCPDTBT ( $\eta_{\mathrm{CD}}=\sim 0.7$ at best) although the main chain configurations are similar between them. In other words, not only effective conjugation length along the main chain but also interchain packing has critical impact on the charge-carrier generation.

The effective electron-hole separation in the CT state would be increased by such charge delocalization in crystalline phases. As shown in Table 1, high charge dissociation efficiencies are observed for crystalline polymers with a correlation length of $L_{010}>4 \mathrm{~nm}$. Interestingly, this is comparable to the effective Coulomb capture radius estimated by taking into account entropy gain associated with changing from a single exciton to two separated charges as suggested by Clarke and Durrant. ${ }^{2}$ We therefore propose that longer effective electron-hole separation distance due to hole delocalization in crystalline phases would promote dissociation into free carriers, resulting in highly efficient charge-carrier generation. This picture is consistent with energetic view of charge-carrier generation from energetically hot states. ${ }^{2,9,60}$ As is discussed in previous reports, the wave function of the hot states are more delocalized compared to the relaxed state, resulting in suppression of Coulomb attraction. ${ }^{61}$
We should discuss the importance of PCBM cluster formation, which has been recently reported by several groups, ${ }^{62,63}$ because crystallization of donor polymers might promote formation of PCBM clusters. As mentioned above, electron delocalization in fullerene clusters would also increase the effective electron-hole separation and hence enhance the charge dissociation efficiency. Furthermore, as suggested by Gregg, ${ }^{64}$ the entropy gain is larger for the charge generation in three-dimensional systems such as fullerenes than in one or two-dimensional systems such as crystalline polymers. We therefore speculate that both hole and electron delocalizations would have positive impact on charge dissociation. In other words, crystalline polymers may have two advantages for free charge generation: hole delocalization over polymer crystalline phase, and electron delocalization over PCBM clusters stimulated by phase segregation.

We further note that recent studies have shown that even allpolymer solar cells exhibit a high PCE of more than 4 and 5\% with high external quantum efficiency. ${ }^{65,66}$ These results suggest that not only electron delocalization in PCBM clusters is a required condition for good charge generation but also charge delocalization in polymers is. We therefore propose that both of hole delocalization in polymer crystallites and electron delocalization in fullerene clusters play an important role in crystalline polymer solar cells and fullerene clusters play a dominant role especially in amorphous polymer solar cells.

\section{Conclusions}

We have studied charge generation and recombination dynamics in PSBTBT/PCBM blend films by transient absorption spectroscopy. Upon photoexcitation of the PSBTBT absorption band at $800 \mathrm{~nm}$, PSBTBT singlet excitons were promptly generated, and then rapidly converted into polarons in disorder phase and polarons in crystalline phase. The prompt polaron formation is attributed to PSBTBT small crystalline domains, which may be comparable to PSBTBT singlet excitons. Polarons are dissociated into free carriers in crystalline phase with almost $100 \%$ efficiency. On the other hand, only a half of polarons are dissociated into free carriers in disorder phase and the others deactivate geminately to the ground state. Interestingly, a part of polarons are transferred from disorder to crystalline phase because of the potential energy cascade between them. As a result, PSBTBT/PCBM blend films exhibit relatively high charge-carrier generation efficiency of $\sim 0.75$. With more efficient hole transfer, the overall charge dissociation efficiency would be further improved. We speculate that polymer crystallizations promote not only hole delocalization over polymer crystalline phase but also electron delocalization over PCBM clusters stimulated by phase segregation. These findings provide in depth understanding of the role of polymer crystallinity on the efficient charge-carrier generation in organic solar cells.

\section{Experimental}

\section{Sample Preparation}

PSBTBT was purchased from Solarmer Materials Inc. with a weight-averaged molecular weight of $M_{\mathrm{w}}=1.7 \times 10^{4}$, and a polydispersity of 2.0, and used without further purification. PCBM was purchased from Frontier Carbon (E-100H), and used without further purification. Thin films were prepared by spincoating from an $\boldsymbol{O}$-dichlorobenzene or chloroform solution 
on glass substrates. The film thickness was about $100-200$ nm.

\section{Measurements}

UV-visible absorption spectra were measured with a UV-visible spectrophotometer (Hitachi, U-3500). Femtosecond transient absorption data were collected with a pump and probe femtosecond transient spectroscopy system. This system consists of a transient absorption spectrometer (Ultrafast Systems, Helios) and a regenerative amplified Ti:sapphire laser (Spectra-Physics, Hurricane). The excitation pulse was set at $800 \mathrm{~nm}$ with a spot size of $\sim 1 \mathrm{~mm}^{2}$. The excitation Microsecond transient absorption data were collected with a highly sensitive microsecond transient absorption system. The excitation pulse was set at $400 \mathrm{~nm}$ with a spot size of $\sim 1 \mathrm{~cm}^{2}$. The sample films were sealed in a quartz cuvette purged with $\mathrm{N}_{2}$. Note that the transient absorption spectra and dynamics were highly reproducible even after the several times measurements. In other words, the laser irradiation had negligible effects on the sample degradation at least under this experimental condition. Details of transient absorption measurements are described in the Supplementary Information.

\section{Acknowledgements}

This work was partly supported by JST PRESTO program (Photoenergy and Conversion Systems and Materials for the Next-Generation Solar Cells).

\section{Notes and references}

${ }^{a}$ Department of Polymer Chemistry, Graduate School of Engineering, Kyoto University, Katsura, Nishikyo, Kyoto 615-8510, Japan.

${ }^{b}$ Japan Science and Technology Agency (JST), PRESTO, 4-1-8 Honcho Kawaguchi, Saitama 332-0012, Japan.

Electronic Supplementary Information (ESI) available: [Experimental details of transient absorption measurements, photoluminescence spectra of PSBTBT pristine and PSBTBT/PCBM blend films, electroluminescence spectrum of a PSBTBT/PCBM blend film, photoluminescence decay measurement, ionization potential measurements, transient absorption spectra of PSBTBT in a 1chloronaphthalene solution, assignments of transient absorption spectra, details of spectral simulation]. See DOI: 10.1039/b000000x/

1. B. Kippelen and J.-L. Brédas, Energy Environ. Sci., 2009, 2, 251.

2. T. M. Clarke and J. R. Durrant, Chem. Rev., 2010, 110, 6736.

3. C. Deibel and V. Dyakonov, Rep. Prog. Phys., 2010, 73, 096401.

4. A. J. Heeger, Chem. Soc. Rev., 2010, 39, 2354.

5. J. Nelson, Mater. Today, 2011, 14, 462.

6. H. Ohkita and S. Ito, Polymer, 2011, 52, 4397.

7. G. Li, R. Zhu and Y. Yang, Nat. Photonics, 2012, 6, 153.

8. L. Dou, J. You, Z. Hong, Z. Xu, G. Li, R. A. Street and Y. Yang, Adv. Mater., 2013, 25, 6642.

9. S. D. Dimitrov and J. R. Durrant, Chem. Mater., 2014, 26, 616.

10. J. Guo, H. Ohkita, H. Benten and S. Ito, J. Am. Chem. Soc., 2010, 132, 6154.

11. I. A. Howard, R. Mauer, M. Meister and F. Laquai, J. Am. Chem. Soc., 2010, 132, 14866.

12. F. Etzold, I. A. Howard, N. Forler, D. M. Cho, M. Meister, H Mangold, J. Shu, M. R. Hansen, K. Müllen and F. Laquai, J. Am. Chem. Soc., 2012, 134, 10569.

13. S. Shoaee, S. Subramaniyan, H. Xin, C. Keiderling, P. S. Tuladhar, F. Jamieson, S. A. Jenekhe and J. R. Durrant, Adv. Funct. Mater., 2013, 23, 3286.

14. M. Scarongella, A. A. Paraecattil, E. Buchaca-Domingo, J. D. Douglas, S. Beaupré, T. McCarthy-Ward, M. Heeney, J.-E. Moser, M. Leclerc, J. M. J. Fréchet, N. Stingelin and N. Banerji, J. Mater. Chem. A, 2014, 2, 6218.
15. S. Hugger, R. Thomann, T. Heinzel and T. Thurn-Albrecht, Colloid. Polym. Sci., 2004, 282, 932.

16. J. Guo, H. Ohkita, H. Benten and S. Ito, J. Am. Chem. Soc., 2009, 131, 16869.

17. M. Campoy-Quiles, Y. Kanai, A. El-Basaty, H. Sakai and H. Murata, Org. Electron., 2009, 10, 1120

18. B. Grévin, R. Rannou, R. Payerne, A. Pron and J. P. Travers, J. Chem. Phys., 2003, 118, 7097.

19. Y. Kim, S. Cook, S. M. Tuladhar, S. A. Choulis, J. Nelson, J. R. Durrant, D. D. C. Bradley, M. Giles, I. Mcculloch, C.-S. Ha and M. A. Ree, Nat. Mater., 2006, 5, 197.

20. A. Salleo, R. J. Kline, D. M. DeLongchamp and M. L. Chabinyc, Adv. Mater., 2010, 22, 3812.

21. D. Chen, A. Nakahara, D. Wei, D. Nordlund and T. P. Russell, Nano. Lett., 2011, 11, 561.

22. D. E. Motaung, G. F. Malgas and C. J. Arendse, J. Mater. Sci., 2010, 45, 3276.

23. J. Y. Kim, S. H. Kim, H.-H. Lee, K. Lee, W. Ma, X. Gong and A. J. Heeger, Adv. Mater., 2006, 18, 572.

24. M. D. Irwin, D. B. Buchholz, A. W. Hains, R. P. H. Chang and T. J. Marks, PNAS, 2008, 105, 2783.

25. G. Dennler, M. C. Scharber and C. J. Brabec, Adv. Mater., 2009, 21, 1323.

26. D. Mühlbacher, M. Scharber, M. Morana, Z. Zhu, D. Waller, R. Gaudiana and C. Brabec, Adv. Mater., 2006, 18, 2884.

27. J. Peet, J. Y. Kim, N. E. Coates, W. L. Ma, D. Moses, A. J. Heeger and G. C. Bazan, Nat. Mater., 2007, 6, 497.

28. J. K. Lee, W. L. Ma, C. J. Brabec, J. Yuen, J. S. Moon, J. Y. Kim, K. Lee, G. C. Bazan and A. J. Heeger, J. Am. Chem. Soc., 2008, 130, 3619.

29. J. Hou, H.-Y. Chen, S. Zhang, G. Li and Y. Yang, J. Am. Chem. Soc., 2008, 130, 16144.

30. M. Morana, H. Azimi, C. Dennler, H.-J. Egelhaaf, M. Scharber, K. Forberich, J. Hauch, R. Gaudiana, D. Waller, Z. Zhu, K. Hingerl, S. S. van Bavel, J. Loos and C. J. Brabec, Adv. Funct. Mater., 2010, 20, 1180 .

31. H.-Y. Chen, J. Hou, A. E. Hayden, H. Yang, K. N. Houk and Y. Yang, Adv. Mater., 2010, 22, 371.

32. M. C. Scharber, M. Koppe, J. Gao, F. Cordella, M. A. Loi, P. Denk, M. Morana, H.-J. Egelhaaf, K. Forberich, G. Dennler, R. Gaudiana, D. Waller, Z. Zhu, X. Shi and C. J. Brabec, Adv. Mater., 2010, 22, 367.

33. H. Lu, B. Akgun and T. P. Russell, Adv. Energy Mater, 2011, 1, 870.

34. H. Lv, X. Zhao, W. Xu. H. Li, J. Chen and X. Yang, Org. Electron., 2013, 14, 1874

35. M. Koppe, H.-J. Egelhaaf, E. Clodic, M. Morana, L. Lüer, A. Troeger, V. Sgobba, D. M. Guldi, T. Ameri and C. J. Brabec, Adv. Energy Mater., 2013, 3, 949.

36. R. Österbacka, C. P. An, X. M. Jiang and Z. V. Vardeny, Sience, 2000, 287, 839.

37. X. M. Jiang, R. Österbacka, O. J. Korovyanko, C. P. An, B. Horovitz, R. A. J. Janssen and Z. V. Vardeny, Adv. Funct. Mater, 2002, 12, 587.

38. S. Yamamoto, J. Guo, H. Ohkita and S. Ito, Adv. Funct. Mater, 2008, 18, 2555.

39. E. R. Malinowski, Factor Analysis in Chemistry 2nd edn., WileyInterscience, John Wiley \& Sons, Inc., New York, U.S.A., 1991.

40. H. Gampp, M. Maeder, C. J. Meyer and A. D. Zuberbühler, Talanta., 1985, 32, 95.

41. S. Yamamoto, H. Ohkita, H. Benten and S. Ito, J. Phys. Chem. C, 2012, 116, 14804

42. A. A. Y. Guilbert, J. M. Frost, T. Agostinelli, E. Pires, S. Lilliu, J. E. Macdonald and J. Nelson, Chem. Mater., 2014, 26, 1226.

43. T. Agostinelli, T. A. M. Ferenczi, E. Pires, S. Foster, A. Maurano, C. Müller, A. Ballantyne, M. Hampton, S. Lilliu, M. Campoy-Quiles, H. Azimi, M. Morana, D. D. C. Bradley, J. Durrant, J. E. Macdonald, N. Stingelin and J. Nelson, J. Polym. Sci. B, Polym. Phys., 2011, 49, 717.

44. S. Honda, S. Yokoya, H. Ohkita, H. Benten and S. Ito, J. Phys. Chem. C, 2011, 115, 11306

45. Assuming a density of the blend film as $1 \mathrm{~g} \mathrm{~cm}^{-3}$ for simplicity, the number density of PCBM molecules is estimated to be $3.3 \times 10^{20} \mathrm{~cm}^{-3}$ at a PCBM fraction of $50 \mathrm{wt} \%$, which corresponds to an average center-to-center distance between neighboring PCBM molecules to be about $0.9 \mathrm{~nm}$ 
46. Y. Tamai, Y. Matsuura, H. Ohkita, H. Benten and S. Ito, J. Phys. Chem. Lett., 2014, 5, 399.

47. K. Chen, A. J. Barker, M. E. Reish, K. C. Gordon and J. M. Hodgkiss, J. Am. Chem. Soc., 2013, 135, 18502.

48. A. A. Paraecattil and N. Banerji, J. Am. Chem. Soc., 2014, 136, 1472.

49. C. Groves, Energy Environ. Sci., 2013, 6, 1546.

50. T. M. Burke and M. D. McGehee, Adv. Mater., 2014, 26, 1923.

51. W. C. Tsoi, S. J. Spencer, L. Yang, A. M. Ballantyne, P. G. Nicholson, A. Turnbull, A. G. Shard, C. E. Murphy, D. D. C. Bradley, J. Nelson and J.-S. Kim, Macromolecules, 2011, 44, 2944.

52. L. Onsager, J. Chem. Phys., 1934, 2, 599.

53. C. L. Braun, J. Chem. Phys., 1984, 80, 4157.

54. M. Wojcik and M. Tachiya, Radiat. Phys. Chem., 2005, 74, 132.

55. P. Peumans and S. R. Forrest, Chem. Phys. Lett., 2004, 398, 27.

56. C. Deibel, T. Strobel and V. Dyakonov, Phys. Rev. Lett., 2009, 103, 036402.

57. S. D. Baranovskii, M. Wiemer, A. V. Nenashev, F. Jansson and F. Gebhard, J. Phys. Chem. Lett., 2012, 3, 1214.

58. D. Caruso and A. Troisi, PNAS, 2012, 109, 13498.

59. P. K. Nayak, K. L. Narasimhan and D. Cahen, J. Phys. Chem. Lett., 2013, 4, 1707.

60. H. Ohkita, S. Cook, Y. Astuti, W. Duffy, S. Tierney, W. Zhang, M. Heeney, I. McCulloch, J. Nelson, D. D. C. Bradley and J. R. Durrant, J. Am. Chem. Soc., 2008, 130, 3030.

61. A. A. Bakulin, A. Rao, V. G. Pavelyev, P. H. M. van Loosdrecht, M. S. Pshenichnikov, D. Niedzialek, J. Cornil, D. Beljonne and R. H. Friend, Science, 2012, 335, 1340.

62. S. Gélinas, A. Rao, A. Kumar, S. L. Smith, A. W. Chin, J. Clark, T. S. van der Poll, G. C. Bazan and R. H. Friend, Science, 2014, 343, 512.

63. B. M. Savoie, A. Rao, A. A. Bakulin, S. Gelinas, B. Movaghar, R. H. Friend, T. J. Marks and M. A. Ratner, J. Am. Chem. Soc., 2014, 136, 2876.

64. B. A. Gregg, J. Phys. Chem. Lett., 2011, 2, 3013.

65. D. Mori, H. Benten, I. Okada, H. Ohkita and S. Ito, Adv. Energy Mater., 2014, 4, 1301006.

66. D. Mori, H. Benten, I. Okada, H. Ohkita and S. Ito, Energy Environ. Sci., submitted. 
Table of Contents artwork

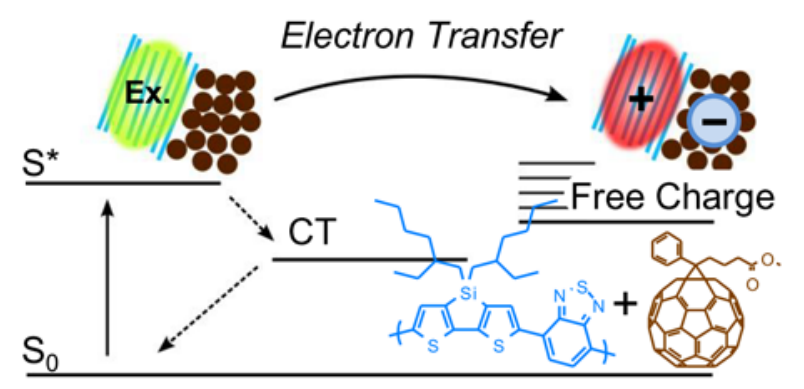

Crystalline donor polymers can promote delocalization of hole polarons and hence result in highly efficient charge-carrier generation in organic solar cells. 